\title{
Property of Solutions to a Biological Population Model with Nonlocal Source and Nonlinear Boundary Condition
}

\author{
Angyang Liu, ${ }^{1, *}$ \\ ${ }^{1}$ School of Mathematical Sciences, University of Science and Technology of China, Anhui Hefei, 230026, China \\ *Corresponding author
}

\begin{abstract}
This paper investigates the property of positive solution to a biological population model with nonlinear boundary condition. Firstly the author gets the local existence result by a regularization method. Then under appropriate hypotheses, the author establishes that positive weak solution either exists globally or blows up in finite time by using comparison principle.
\end{abstract}

Keywords-biological population model; nonlocal source; GLOBAL existence; blow-up; comparison principle

\section{INTRODUCTION}

In this paper, we study the following parabolic equation arising in the diffusion of biological populations with nonlocal source and nonlinear absorption:

$$
\left\{\begin{array}{lc}
u_{t}-\left(\left|u_{x}\right|^{p-2} u_{x}\right)_{x}=\int_{0}^{a} u^{\alpha}(\xi, t) d \xi+k u^{\beta}(x, t), & (x, t) \in[0, a] \times(0, T) \\
\left.u_{x}\right|_{x=0}=0,\left.u_{x}\right|_{x=a}=\left.u^{q}\right|_{x=a}, & t \in(0, T), \\
u(x, 0)=u_{0}(x), & x \in[0, a],
\end{array}\right.
$$

$$
\text { in which } T>0, \quad p>2, \quad \alpha, \beta, k, q>0 \text {, }
$$
$u_{0}(x) \in L^{\infty}(\Omega) \cap W_{0}^{1, p}(\Omega)$. Problems of this form arise in mathematical models such as modeling gas or fluid flow through a porous medium and completely turbulent flow and for the spread of certain biological populations ( see [1,2] and the references therein). Biologists believe that dispersal or emigration play a key role in the regulation of population of some species. Parabolic equations involving a nonlocal source, which arise in a population model that communicates through chemical means, were studied in [3, 4]. The nonlinear boundary condition in (1.1) can be physically interpreted as a nonlinear radiation law, see [5].

Over the last few years, much effort has been devoted to the study of blow-up properties for nonlocal semilinear parabolic equations with nonlinear boundary conditions. Conditions on blowing up, blow-up set, blow-up rate and asymptotic behavior of solutions are obtained (see $[6,7,13,14]$ and the references therein). The problems concerning (1.1) include the existence and multiplicity of global solutions, blowing-up, blow-up rates and blow-up sets, uniqueness and nonuniqueness etc.
A few authors (see $[8,9])$ have investigated the following equation:

$$
u_{t}-\operatorname{div}\left(|\nabla u|^{p-2} \nabla u\right)=u^{q}
$$

with initial and boundary conditions. Roughly speaking, their results are:

(1) the solution $u$ exists globally if $q<p-1$,

(2) $u$ blows up in finite time if $q>p-1$ and $u_{0}(x)$ is sufficiently large.

However, to the author's best knowledge, there is little literature on the study of the global existence and blow-up properties for the system (1.1). Motivated by the above works, in this paper, we study global existence or blow-up of weak solutions to (1.1). Note that (1.1) has nonlinear and nonlocal source $\int_{0}^{a} u^{\alpha}(\xi, t) d \xi$, local term $u^{\beta}(x, t)$ and nonlinear boundary condition $u^{q}$, which make the behavior of the solution different from that for that of homogeneous Neumann or Dirichlet boundary value problems. To overcome these difficulties, we used some modification of the technique in [10] so that we can handle the nonlinearities. In this paper, the blowup means that there exists a $T^{*}<+\infty$ such that $\|u(\cdot, t)\|_{\infty}<\infty \quad$ for $\quad t \in\left(0, T^{*}\right) \quad$ and $\lim _{t \rightarrow T^{*}}\|u(\cdot, t)\|_{\infty}=\infty$.

The outline of this paper is as follows: in the next section, we will prove the local existence results by a regularization method. We will give the proof of a weak comparison principle and discuss the global existence and blow-up of solutions in the third section.

\section{LOCAL EXISTENCE}

In this section, we study the local existence of (1.1) under appropriate hypotheses. From the point of physics, we need only to consider the nonnegative solutions. Since (1.1) is the degenerate parabolic equations for $\left|u_{x}\right|=0$, one can not expect the existence of classical solution. As it is now well 
known that degenerate equations need not posses classical solutions, most of studies of p-Laplacian equations concerned with weak solutions (see $[3,4])$.We begin by giving a precise definition of a weak solution for problem (1.1).

Before stating our main results, we make some assumptions. Let $D=(0, a)$ and $\Omega_{r}=D \times(0, r]$. Let $\bar{D}$ and $\bar{\Omega}_{r}$ be their respective closures, $Z^{+}=\max \{z, 0\}$. Before stating our main results, we make some assumptions on the initial value $u_{0}(x)$.

$\left(H_{1}\right) u_{0}(x) \in C^{2+\alpha}([0, a])$ for some $0<\alpha<1$,

$u_{0}(x) \geq \delta>0$

$\left(H_{2}\right)\left(\left|u_{0 x}\right|^{p-1} u_{0 x}\right)_{x} \in L^{2}([0, a])$;

$\left(H_{3}\right) \quad u_{0}(x)$ satisfies the compatibility conditions: $u_{0 x}(0)=0, u_{0 x}(a)=u_{0}^{q}(a)$.

Definition 2.1. A function $u \in C\left(\Omega_{T}\right)$ is called a supersolution (subsolution) of problem (1.1) in $\Omega_{T}$ if all of the following hold:

(i) $u \in L^{\infty}\left(0, T ; W^{1, \infty}(0, a)\right) \cap W^{1,2}\left(0, T ; L^{2}(0, a)\right)$, $u(x, 0) \geq(\leq) u_{0}(x)$;

(ii) For any nonnegative functions

$\varphi \in L^{1}\left(0, T ; W^{1,2}(0, a)\right) \cap L^{2}\left(\Omega_{T}\right)$,

$\iint_{\Omega_{T}} u_{t}(x, t) \varphi(x, t) d x d t+\iint_{\Omega_{T}}\left|u_{x}(x, t)\right|^{p-2} u_{x}(x, t) \varphi_{x}(x, t) d x d t$

$\geq(\leq) \int_{0}^{T} u^{q}(a, t) \varphi(a, t) d t+\iint_{\Omega_{T}}\left(\int_{0}^{a} u^{\alpha}(\xi, t) d \xi\right) \varphi(x, t) d x d t$

$+k \iint_{\Omega_{T}} u^{\beta}(x, t) \varphi(x, t) d x d t$.

A weak solution of (1.1) is a vector function which is both a subsolution and a supersolution of (1.1). For every $T<\infty$, if $u$ is a solution of (1.1), we say $u$ is global.

Remark 2.2. Clearly, every nonnegative classical (sub-, super-) solution of (1.1) is a weak (sub-, super-) solution of (1.1) in the sense of Definition 2.1.

Theorem 2.3. Assume $\left(H_{1}\right)-\left(H_{3}\right)$, then there exists a $T_{0}>0$ such that (1.1) admits a solution $u \in C\left(0, T_{0} ; L^{\infty}\left(\Omega_{T}\right)\right) \cap L^{p}\left(0, T_{0} ; W_{0}^{1, p}\left(\Omega_{T}\right)\right) \quad$ satisfying $u \geq \delta$ for some positive constant $\delta$. Moreover, if $T<+\infty$, then $\lim _{t \rightarrow T^{-}} \sup _{x \in[0, a]}\|u(\cdot, t)\|_{L^{\infty}}=+\infty$. In this case, we say that the solution $u$ blows up in finite time.
Proof. The proof of this theorem basically follows line by line the proof of Theorem 1 in [10]. Consider the following approximate problems for (1.1):

$$
\left\{\begin{array}{lc}
u_{\varepsilon t}-\left(\left(\left|u_{\varepsilon x}\right|^{2}+\varepsilon\right)^{\frac{p-2}{2}} u_{\varepsilon x}\right)_{x}=F\left(u_{\varepsilon}\right),(x, t) \in(0, a) \times(0, T), \\
\left.u_{\varepsilon x}\right|_{x=0}=0,\left.u_{\varepsilon x}\right|_{x=a}=G\left(u_{\varepsilon}\right), & t \in(0, T), \\
u_{\varepsilon}(x, 0)=u_{0}(x), & x \in[0, a] .
\end{array}\right.
$$

We need to control the nonlocal term by applying the technique developed in [10]. Choose the bounded functions: $F(z), G(z) \in C^{\infty}(R)$ such that:

$$
F(z)=\int_{0}^{a} z^{\alpha}(\xi, t) d \xi-k z^{\beta}(x, t), G(z)=z^{q}
$$

for $\delta \leq z \leq M+1$ where $M=\left\|u_{0}(x)\right\|_{\infty}$. And we assume that there exist positive constants $l$ and $L$ such that $0<l \leq F(z), G(z) \leq L<+\infty, \frac{\partial G(z)}{\partial z} \geq 0$ for any $z \in R$.

Equation (2.2) are a nondegenerate problem for each fixed $\varepsilon$. We divide our proof into four steps.

Step 1. There exists a small constant $t_{1}>0$ and a positive constant $C$ independent of $\varepsilon$ such that:

$$
\left\|u_{\varepsilon X}\right\|_{\infty} \leq C \text { on } \bar{\Omega}_{t_{1}} \text {. }
$$

To this end, choose bounded functions: $\Phi_{\varepsilon}(z) \in C^{\infty}(R), 0<\rho_{\varepsilon} \leq \Phi_{\varepsilon^{\prime}}(z) \leq \rho_{\varepsilon}^{-1}$ and

$\Phi_{\varepsilon}(z)=\left(|z|^{2}+\varepsilon\right)^{\frac{p-2}{2}} z$ for $|z| \leq K+L+1$, where $K$ will be determined later. Then consider the following problem:

$\left\{\begin{array}{lc}u_{\varepsilon t}-\left(\Phi_{\varepsilon}\left(u_{\varepsilon x}\right)\right)_{x}=F\left(u_{\varepsilon}\right), & (x, t) \in(0, a) \times(0, T), \\ \left.u_{\varepsilon x}\right|_{x=0}=0,\left.u_{\varepsilon x}\right|_{x=a}=G\left(u_{\varepsilon}\right), & t \in(0, T), \\ u_{\varepsilon}(x, 0)=u_{0}(x), & x \in[0, a] .\end{array}\right.$

For (2.4), standard parabolic theory (see [11]) shows that there is a solution $u_{\varepsilon}$ in the class $H^{2+\beta, 1+\beta / 2}\left(\bar{\Omega}_{T}\right)$ for some $\beta \in(0,1)$. Obviously, Comparison principle holds for (2.4). Therefore,

$u_{\varepsilon}(x, t) \geq \delta>0, \quad u_{\varepsilon t}(x, t) \geq 0, \quad(x, t) \in \bar{\Omega}_{T}$ and for some constant $c \in R, 0<c \leq F^{\prime}(z) \leq c^{-1}$.

Similarly to the proof of Proposition 3.1 in [10], there exists a small constant $t_{1}>0$ such that: 
$\left\|u_{\varepsilon x}\right\|_{\infty} \leq K+L+1$ on $\bar{\Omega}_{t_{1}}$, where $K=\left\|u_{0 x}(x)\right\|_{\infty}$. Thus $u_{\varepsilon}$ is a solution of (2.2) in $\bar{\Omega}_{t_{1}}$. Setting $C=K+L+1$, we get the conclusion.

Step 2. There exist a constant $t_{2}>0$, such that $u_{\varepsilon}(x, t) \leq M+1$ on $\bar{\Omega}_{t_{2}}$. We may assume that $T \in[0,1)$. Let $h \geq M$. In fact, multiplying (2.2) by $\left(u_{\varepsilon}-h\right)^{+}$and integrating over $\bar{\Omega}_{T}$, we obtain

$$
\begin{aligned}
& \int_{0}^{a}\left(u_{\varepsilon}-h\right)^{+2} d x+\iint_{\bar{\Omega}_{T}}\left|u_{\varepsilon x}\right|^{p-2}\left(u_{\varepsilon}-h\right)_{x}^{+2} d x d t \leq \\
& \left.c \int_{0}^{T}\left(u_{\varepsilon}-h\right)^{+}\right|_{x=a} d t+c \iint_{\bar{\Omega}_{T}}\left(u_{\varepsilon}-h\right)^{+} d x d t
\end{aligned}
$$

for some positive constant $C$ independent of $\varepsilon$. Then similarly to the proof of Proposition 3.1 in [10], there exists a $t_{2}>0$, independent of $\varepsilon$, such that $u_{\varepsilon}(x, t) \leq M+1$ on $\bar{\Omega}_{t_{2}}$.

Step 3. There exist constant $M_{1}$, independent of $\varepsilon$, such that $\left|u_{s t}\right|_{L^{2}\left(\bar{\Omega}_{T}\right)} \leq M_{1}<+\infty$, where $T=\min \left\{t_{1}, t_{2}\right\}$.To do so, multiplying (2.2) by $u_{\varepsilon t}$ and integrating over $\bar{\Omega}_{T}$, we have

$$
\begin{aligned}
& \frac{1}{2} \int_{0}^{a} u_{\varepsilon t}^{2} d x+\iint_{\bar{\Omega}_{T}}\left(u_{\varepsilon x}^{2}+\varepsilon\right)^{(p-2) / 2-1}\left(p u_{\varepsilon x}^{2}+\varepsilon\right) u_{\varepsilon t}^{2} d x d t \\
& =\frac{1}{2} \int_{0}^{a} u_{\varepsilon 0 t}^{2} d x+\int_{0}^{T}\left(\left(u_{\varepsilon x}^{2}+\varepsilon\right)^{(p-2) / 2-1} u_{\varepsilon x}\right)_{t} u_{\varepsilon t}(a, t) d t \\
& +\iint_{\bar{\Omega}_{T}} F^{\prime} u_{\varepsilon t}^{2} d x d t
\end{aligned}
$$

Similarly to the proof of Proposition 3.1 in [19], we have

$$
\int_{0}^{a} u_{\varepsilon t}^{2} d x \leq \int_{0}^{a} u_{\varepsilon 0 t}^{2} d x+C \iint_{\bar{\Omega}_{T}} u_{\varepsilon t}^{2} d x d t+C \iint_{\bar{\Omega}_{T}} F^{\prime} u_{\varepsilon t}^{2} d x d t
$$

Using Young's inequality, we have

$$
\int_{0}^{a} u_{\varepsilon t}^{2} d x \leq \int_{0}^{a} u_{\varepsilon 0 t}^{2} d x+C \iint_{\bar{\Omega}_{T}} u_{\varepsilon t}^{2} d x d t
$$

By the Gronwall's lemma, we obtain the desired results.

Step 4. Therefore, by the Aubin theorem, it follows that (up to extraction of a subsequence):

$$
\begin{gathered}
u_{\varepsilon} \rightarrow u, \quad \text { a.e. for } \quad(x, t) \in \Omega_{T}, \\
u_{\varepsilon x} \rightarrow u_{x}, \quad \text { weakly in } L^{\infty}\left(\Omega_{T}\right),
\end{gathered}
$$

$$
\begin{gathered}
u_{s t} \rightarrow u_{t}, \quad v_{n t} \rightarrow v_{t}, \quad \text { weakly in } L^{2}\left(\Omega_{T}\right), \\
\Phi_{\varepsilon}\left(u_{\varepsilon x}\right)_{x_{i}} \rightarrow\left|u_{x}\right|^{p-2} u_{x} \text { weakly in } L^{\infty}\left(\Omega_{T}\right) .
\end{gathered}
$$

From (2.3), we have

$$
\lim _{n \rightarrow \infty} \iint_{\Omega_{T}} \psi\left|u_{\varepsilon x}\right|^{p-2} u_{\varepsilon x}\left(u_{\varepsilon}-u\right)_{x} d x d t=0
$$

where $\psi \in C_{0}^{1,1}\left(\Omega_{T}\right), \psi \geq 0$. Then similarly to the proof of Theorem 2.1 in [12], we have

$$
\iint_{\Omega_{T}} \psi\left(\left|u_{x}\right|^{p-2} u_{x}-\left|u_{\varepsilon x}\right|^{p-2} u_{\varepsilon x}\right) d x d t=0 .
$$

The proof of Theorem 2.3 is completed by a standard limiting process.

\section{GLOBAL EXISTENCE AND BLOW-UP}

In this section, we shall discuss the global existence and blow-up in finite time of the solution for system (1.1). Throughout this section we denote $\tau=\frac{p-1}{p}$ and choose $\bar{\lambda}, \underline{\lambda}$ satisfying $\bar{\lambda}>1>\underline{\lambda}>0$. Our approach in a combination principle and upper and sub-technique. In order to prove our results, we give the following weak comparison principle.

Lemma 3.1 (Comparison principle). Assume that $u$ be a weak solution of (1.1) in $\Omega_{T}, \underline{u} \geq \delta$ and $\bar{u}$ a subsolution and a supersolution of (1.1) in $\Omega_{T}$, respectively, with nonlinear boundary flux $\underline{\lambda} \underline{u}^{q}, \bar{\lambda} \bar{u}^{q}$ and nonlocal terms $\underline{\lambda}\left(\int_{0}^{a} \underline{u}^{\alpha}(\xi, t) d \xi+k \underline{u}^{\beta}(x, t)\right) \quad, \quad \bar{\lambda}\left(\int_{0}^{a} \bar{u}^{\alpha}(\xi, t) d \xi+k \bar{u}^{\beta}(x, t)\right)$. Then $\underline{u} \leq u \leq \bar{u}$ on $\bar{\Omega}_{T}$.

Theorem 3.2. If $\alpha<1,(1-\alpha)(q-1)>0$, then the solution of (1.1) blows up in finite time.

Proof. It is easy to prove that there exist positive constants $m_{1}>0, m_{2}>1$ satisfying

$$
m_{1} m_{2}+1-\alpha=0, \quad m_{1} m_{2}-m_{1}-m_{1} m_{2} q \leq 0 .
$$

Set $\quad w_{1}=\left[d\left(1+x^{\frac{1}{\tau}}\right)+(c-b t)^{-m_{1}}\right]^{m_{2}}=\left[S_{1}\right]^{m_{2}}, \quad$ where $b, c, d>0$ satisfy

$$
b \leq \underline{\lambda} a\left(m_{1} m_{2}\right)^{-1} 2^{1-m_{2}}, \quad c \geq 2^{\frac{1}{m_{1}}} \delta^{-\frac{1}{m_{1} m_{2}}},
$$




$$
d \leq \min \left\{\frac{1}{1+a^{\frac{1}{\tau}}} c^{-m_{1}}, \underline{\lambda}\left(m_{2} \frac{1}{\tau} a^{\frac{1}{p-1}} 2^{m_{2}-1}\right)^{-1} c^{m_{1} m_{2}-m_{1}-m_{1} m_{2} q}\right\} .
$$

Computing directly, we obtain

$w_{1 t}=m_{2}\left[S_{1}\right]^{m_{2}-1} m_{1} b(c-b t)^{-m_{1}-1}, w_{1 x}=m_{2}\left[S_{1}\right]^{m_{2}-1} d \frac{1}{\tau} x^{\frac{1}{p-1}}$,

We have

$$
w_{1 t} \leq\left(w_{1 x}^{p-1}\right)_{x}+\lambda\left(\int_{0}^{a} w_{1}^{\alpha}(\xi, t) d \xi+k w_{1}^{\beta}\right) .
$$

Noting that on the boundary

$$
\begin{aligned}
& w_{1 x}(a, t)=m_{2}\left[S_{1}\right]^{m_{2}-1} d \frac{1}{\tau} a^{\frac{1}{p-1}} \leq \\
& m_{2} d \frac{1}{\tau} a^{\frac{1}{p-1}} 2^{m_{2}-1}(c-b t)^{-m_{1}\left(m_{2}-1\right)} \leq \underline{\lambda} w_{1}^{q} .
\end{aligned}
$$

Under the assumptions of $a, b, m_{1}, m_{2}$, we have that for $x \in[0,1]$,

$$
w_{1}(x, 0) \leq\left[d\left(1+a^{\frac{1}{\tau}}\right)+c^{-m_{1}}\right]^{m_{2}} \leq \delta \leq u_{0}(x) .
$$

From (3.1)-(3.3) and the comparison principle, it follows that $u \geq w_{1}$. This shows that $u$ blows up in finite time.

Theorem 3.3. If $\alpha=\beta \leq 1$ and $u_{0}(x)$ satisfies $\left(H_{1}\right)-\left(H_{3}\right)$. Let $\varphi(x)$ be the unique positive solution of the following linear elliptic problem

$$
\left\{\begin{array}{lr}
-\left(\left|\varphi_{x}\right|^{p-2} \varphi_{x}\right)_{x}=1, & x \in(0, a), \\
\varphi^{\prime}(0)=0, & \varphi^{\prime}(a)=1 .
\end{array}\right.
$$

Then there exists constants $a_{1}>0$ such that the solution $u(x, t)$ of (1.1) exists globally when $u_{0}(x) \leq a_{1} \varphi(x)$.

Proof. Let $w_{2}(x)=a_{1} \varphi(x)$ where $a_{1}>0$ is chosen so that

$$
\begin{aligned}
& -\left(\left|w_{2 x}\right|^{p-2} w_{2 x}\right)_{x}=a_{1}>\bar{\lambda} a_{1}^{m}\left(\int_{0}^{a} \varphi^{\alpha}(\xi) d \xi+k \varphi^{\alpha}(x)\right) \\
& =\bar{\lambda}\left(\int_{0}^{a} w_{2}^{\alpha}(\xi) d \xi+k w_{2}^{\alpha}(x)\right),
\end{aligned}
$$

$$
\left.w_{2 x}\right|_{x=a}=a_{1} \geq \bar{\lambda} w_{2}^{q},
$$

By Lemma 3.1 it follows that $u(x, t)$ exists globally provided that $u_{0}(x) \leq w_{2}(x)=a_{1} \varphi(x)$.

\section{SUMMARY}

By using comparison principle, we have obtained that the solution of (1.1) either exists globally or blow-up in finite time. To overcome the difficulties, we used some modification of the technique so that we can handle the nonlinearities. In the following work, we will concern the quenching of solutions of (1.1) and the global stability of diffusive predator-prey system in a bounded domain with no-flux boundary condition.

\section{REFERENCES}

[1] G.Astrita and G.Marrucci. Principles of Non-Newtonian Fluid Mechanics, McGraw-Hill, 1974

[2] Escobedo M. and Herrero M.A. A semilinear parabolic system in a bounded domain, Ann. Mat. Pura Appl. 165(1993), 315-336

[3] J. Furter, M. Grinfeld. Local vs. nonlocal interactions in population dynamics, J. Math. Biol. 27(1989), 65-80

[4] J.R. Anderson, K. Deng. Global existence for degenerate parabolic equations with a non-local forcing, Math. Methods Appl. Sci. 20 (1997), 1069-1087

[5] J. Filo. Diffusivity versus absorption though the boundary, J. Differential Equations 99 (2) (1992) 281-305

[6] P. Souplet. Uniform blow-up profiles and boundary behavior for diffusion equations with nonlocal nonlinear source[J], J. Differential Equations, 153(1999), 374-406

[7] M. Wang, Y. Wang. Properties of positive solutions for non-local reaction-diffusion systems. Math. Meth. Appl. Sci. 19(1996), 1141-1156

[8] J. Zhao. Existence and nonexistence of solutions for $u_{f}-\operatorname{div}\left(|\nabla u|^{p-2} \nabla u\right)=f(\nabla u, u, x, t)$, J.Math. Anal. Appl. 172(1993), 130-146.

[9] H. Ishii. Asymptotic stability and blowing up of solutions of some nonlinear equations, J. Differential Equations 26(1977), 291-319.

[10] S. Wang. Doubly nonlinear degenerate parabolic systems with coupled nonlinear boundary conditions, J. Differential Equations 182 (2002) 431469.

[11] O.A. Ladyzenskaja, V.A. Solonnikov, N.N. Uralceva. Linear and Quasilinear Equations of Parabolic Type, Trans. Math. Monogr., Amer. Math. Soc., Providence, RI, 1968.

[12] J. Zhao. Existence and nonexistence of solutions for $u_{t}-\operatorname{div}\left(|\nabla u|^{p-2} \nabla u\right)=f(\nabla u, u, x, t)$, J.Math. Anal. Appl. 172(1993), $130-146$

[13] C.V. Pao. Blowing-up of solution for a nonlocal reaction-diffusion problem in combustion theory. J.Math.Anal.Appl. 166(1992), 591-600.

[14] Z. Cui, Z. Yang. Roles of weight functions to a nonlinear porous medium equation with nonlocal source and nonlocal boundary condition, J. Math. Anal Appl. 342 (2008), 559-570. 